\title{
Communicating a Prognosis: a Randomized Trial of Survival Rate Language
}

\author{
Eric P. Silver, MS ${ }^{l}$, Stephen B. Broomell, $P h D^{7}$, Alexander L. Davis, $P h D^{2}$, \\ Douglas $B$. White, $M D^{3}$, and Tamar Krishnamurti, $P h D^{4}$
}

'Department of Social and Decision Sciences, Carnegie Mellon University, Pittsburgh, PA, USA; ${ }^{2}$ Department of Engineering and Public Policy, Carnegie Mellon University, Pittsburgh, PA, USA; ${ }^{3}$ Departments of Medicine and Critical Care Medicine, University of Pittsburgh School of Medicine, Pittsburgh, PA, USA; ${ }^{4}$ Division of General Internal Medicine, University of Pittsburgh, Pittsburgh, PA, USA.

\section{INTRODUCTION}

As medical practice transitions toward shared decisionmaking between physicians and patients, it is essential that prognostic information is clearly communicated. ${ }^{1}$ Laypeople are able to make reasonable statistical inferences about familiar domains. ${ }^{2}$ However, Kaplan-Meier curves, commonly used by physicians in estimating survival rates over time, are unfamiliar to patients. We test two prognoses, which communicate cumulative mortality and duration, and find lay understandings are more affected by the chosen time period than survival probability when estimating survival duration.

As can be seen in Figure 1, an equivalent survival rate may be communicated to a patient for various time frames (e.g., a 3 -year survival rate of $25 \%$, a 5 -year survival rate of $10 \%$, or a $37 \%$ annual hazard rate), ${ }^{3}$ depending on physician preference. In this study, we tested how prognoses, reflecting the same underlying hazard-equivalent risk, but communicating cumulative mortality at different timepoints, affect lay understanding of survival. Study design and planned analyses were preregistered at the Open Science Framework (OSF).

\section{METHODS}

Participants received $\$ 1$ for completing a survey regarding a hypothetical illness. A total of 560 US respondents were recruited from an online service (Amazon's Mechanical Turk [MTurk]). Of these, 218 failed inclusion criteria (completing the task in $<3 \mathrm{~min}$, passing an attention check, and correctly indicating that the longest duration a person might live was longer than the shortest they might live), leaving 342 valid responses $(55.0 \%$ men; mean age $=42$ years $)$.

We randomly assigned respondents to one of two descriptions of a disease prognosis with an equivalent hazard rate: $25 \%$ over 3 years or $10 \%$ over 5 years. Participants responded to the following prompt:

Published online May 29, 2019
I'm very sorry to tell you this. I've just taken a look at the scans and see evidence that you have a severe disease. This is the sort of thing that some people won't survive. The $(3 \mid 5)$ year survival rate of this disease is (25 10) percent. We don't know exactly what will happen to you, but we do know that $(75 \mid 90)$ percent of patients like you will die in the next $(3 \mid 5)$ years.Participants were then asked for their best guess of (a) how much time would pass before they would die from the disease and (b) how much time would pass before a similar other would die. Responses were provided in number of years, months, and days. We elicited two dependent variables (DVs). DV1 is an aggregate of their best guess of when they would die and when a similar person would die. Each component of DV1 is also individually significant. DV2 is an aggregate of two measures of their uncertainty in their guess. We only report results for DV1, their expected lifespan.

\section{RESULTS}

Using a Wilcoxon rank sum test, the median \pm SD expectation of remaining lifespan was significantly longer in the 5-year condition $(3.0 \pm .83$ years, $N=124)$ than in the 3 -year condition $(2.1 \pm 2.0$ years, $N=136), P<.001$.

\section{DISCUSSION}

We found that judgments of expected survival differed between conditions presenting the same survival curve for different timeframes. Overall, respondents expect to live $43 \%$ longer when evaluating an equivalent prognosis at 5 versus 3 years, despite adjusted survivability estimates (25\% versus $10 \%)$. These results suggest that patients may weight timeframe more heavily than probability of survival when cognitively processing prognoses. As such, a physician's choice of communicated target date could have a strong effect on the perception of expected survival. 


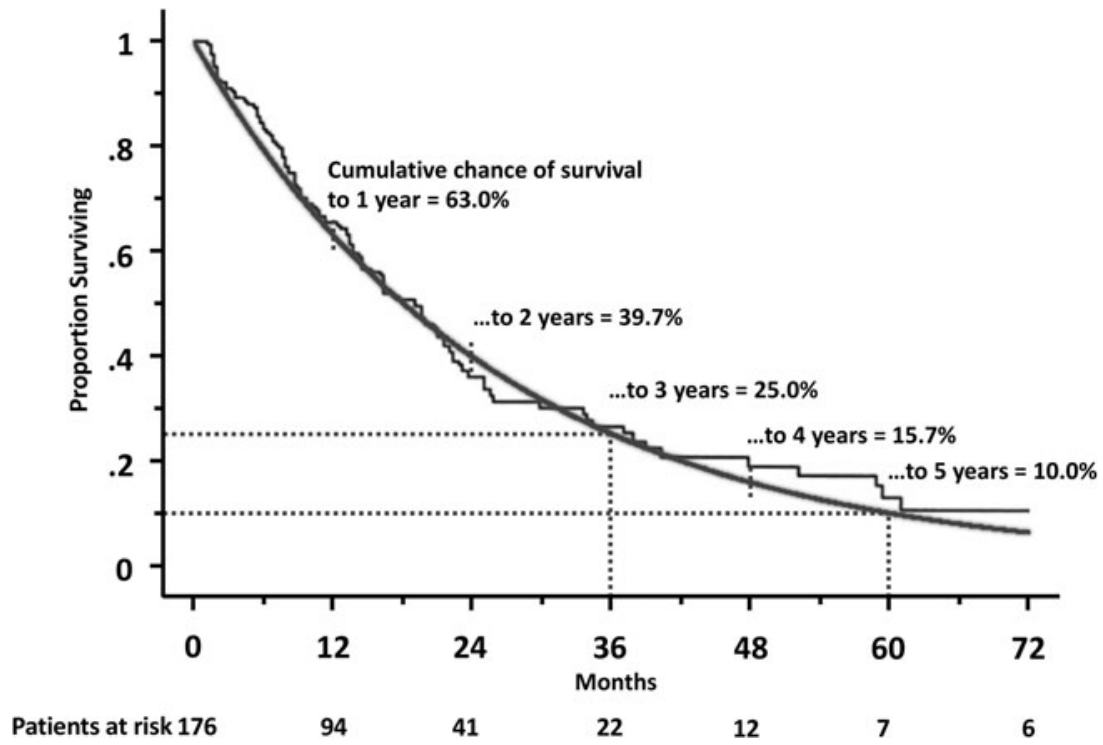

Figure 1 Equivalent mortality risks of pleural mesothelioma per year. Estimated survival distribution for pleural mesothelioma modified from Fig. 1 from Sugarbaker et al. (1999). The curved line represents a 37\% annualized hazard rate with values labeled for years 1 through 5.

Corresponding Author: Tamar Krishnamurti, PhD; Division of General Internal Medicine, University of Pittsburgh, Pittsburgh, PA, USA (e-mail: tamark@pitt.edu).

Funding Information Dr. Krishnamurti's time was financially supported by an Institutional K-award at the University of Pittsburgh (NIH KL2 TRO01856). Funding for data collection was from discretionary internal research funds.

\section{Compliance with Ethical Standards:}

Conflict of Interest: The authors declare that they do not have a conflict of interest.

\section{REFERENCES}

1. Fagerlin A, Zikmund-Fisher BJ, Ubel PA. Helping patients decide: ten steps to better risk communication. J Natl Cancer Inst. 2011;103(19):1436-1443.

2. Griffiths TL, Tenenbaum JB. Optimal predictions in everyday cognition. Psychol Sci. 2006; 17(9):767-773.

3. Sugarbaker DJ, Flores RM, Jaklitsch MT, Richards WG, Strauss GM, Corson JM, ... Baldini EH. Resection margins, extrapleural nodal status, and cell type determine postoperative long-term survival in trimodality therapy of malignant pleural mesothelioma: results in 183 patients. J Thorac Cardiovasc Surg. 1999; 117(1):54-65.

Publisher's Note Springer Nature remains neutral with regard to jurisdictional claims in published maps and institutional affiliations. 